\title{
ARTICLE
}

\section{Revealing the role of organic cations in hybrid halide perovskite $\mathrm{CH}_{3} \mathrm{NH}_{3} \mathrm{Pbl}_{3}$}

Carlo Motta ${ }^{1,2}$, Fedwa El-Mellouhi ${ }^{2}$, Sabre Kais ${ }^{2,3},{\text { Nouar } \text { Tabet }^{2} \text {, Fahhad Alharbi }{ }^{2} \& \text { Stefano Sanvito }}^{1}$

The hybrid halide perovskite $\mathrm{CH}_{3} \mathrm{NH}_{3} \mathrm{Pbl}_{3}$ has enabled solar cells to reach an efficiency of about $20 \%$, demonstrating a pace for improvements with no precedents in the solar energy arena. Despite such explosive progress, the microscopic origin behind the success of such material is still debated, with the role played by the organic cations in the light-harvesting process remaining unclear. Here van der Waals-corrected density functional theory calculations reveal that the orientation of the organic molecules plays a fundamental role in determining the material electronic properties. For instance, if $\mathrm{CH}_{3} \mathrm{NH}_{3}$ orients along a (011)-like direction, the $\mathrm{Pbl}_{6}$ octahedral cage will distort and the bandgap will become indirect. Our results suggest that molecular rotations, with the consequent dynamical change of the band structure, might be at the origin of the slow carrier recombination and the superior conversion efficiency of $\mathrm{CH}_{3} \mathrm{NH}_{3} \mathrm{Pbl}_{3}$.

\footnotetext{
${ }^{1}$ School of Physics, AMBER and CRANN Institute, Trinity College, Dublin Dublin 2, Ireland. ${ }^{2}$ Qatar Environment and Energy Research Institute, PO Box 5825 Doha, Qatar. ${ }^{3}$ Department of Chemistry and Physics, Purdue University, West Lafayette, Indiana 46323, USA. Correspondence and requests for materials should be addressed to C.M. (email: mottac@tcd.ie) or to F.E.-M. (email: felmellouhi@gf.org.qa).
} 
$\mathrm{V}$ ery few materials have taken a research field by storm as the hybrid halide perovskites have done in the last 2 years in the solar cell community ${ }^{1}$. These are compounds with the standard $\mathrm{AMX}_{3}$ perovskite structure, where $\mathrm{X}$ is the halide iodine, $\mathrm{M}$ is lead, while the remaining cation position, $\mathrm{A}$, is taken by an organic molecule, in this case methylammonium (MA), $\mathrm{CH}_{3} \mathrm{NH}_{3}$. The success of such compounds is that high-efficiency solar cells can be fabricated cheaply from the liquid phase $\mathrm{e}^{2,3}$ and that the efficiency can be tuned by controlling their structural order and composition ${ }^{4,5}$. Particularly intriguing is the fact that high efficiencies are achieved even for planar cells ${ }^{6}$, indicating that the charge separation can occur in the hybrid perovskite absorber and that efficient charge diffusion takes place for both electrons and holes ${ }^{7}$.

Part of this behaviour can be related to the unusual situation concerning possible intrinsic defects. Recent electronic structure calculations ${ }^{8,9}$ have shown that the dominant simple or complex defects in $\mathrm{CH}_{3} \mathrm{NH}_{3} \mathrm{PbI}_{3}$ produce only shallow defect levels, while those able to act as deep traps have a high formation energy. Such observation suggests that, under ideal conditions, deep-level point defects cannot act as non-radiative recombination sites-a fact that can partially explain the relative long exciton lifetimes and diffusion lengths observed in these materials. At the same time, the absence of deep levels may explain also the large open-circuit voltage measured. However, despite all these hints, many fundamental questions remain open. In particular, it is still not clear what is the role played by the organic molecules in the efficiency of these compounds. Theoretical investigations have now built a consensus that the MA group does not have any significant contribution to the electronic structure around the band edges ${ }^{8,10,11}$ and suggest that the only role of the molecules is to stabilize electrostatically the perovskite structure.

Interestingly, not even the geometrical arrangement of the MA group is known in detail. At low temperature (below $150 \mathrm{~K}$ ), the crystal adopts an orthorhombic (Pnma) structure, in which the $\mathrm{PbI}_{6}$ octahedra are strongly deformed assuming a rectangular basal plane. Such deformation restricts the rotational degrees of freedom of MA in the rhombus-shaped interstitial region, thus imposing a spatial ordering to $\mathrm{CH}_{3} \mathrm{NH}_{3}$. In this case, the organic cation is pinned and can only rotate along the $\mathrm{C}-\mathrm{N}$ axis. As the temperature is increased and the material progressively assumes a cubic structure $(P m-3 m)$, by passing through a tetragonal one $(\mathrm{I} / \mathrm{m})$, the $\mathrm{CH}_{3} \mathrm{NH}_{3}$ molecules become free to rotate between the octahedral cages. Above room temperature, such rotation is fast, to a point where both crystallographic analysis ${ }^{3,12}$ and NMR measurements ${ }^{13}$ have shown that the exact location of the MA groups cannot be determined.

Here we conduct density functional theory (DFT) calculations for $\mathrm{CH}_{3} \mathrm{NH}_{3} \mathrm{PbI}_{3}$ in the cubic phase, by taking into consideration the experimentally reported evidence of the fast rotation of $\mathrm{CH}_{3} \mathrm{NH}_{3}$. This means that we do not limit our analysis to $\mathrm{CH}_{3} \mathrm{NH}_{3}$ oriented along the (100) or (111) direction, for which a higher symmetry is maintained, but we also explore cases where the symmetry is lowered. We find that such symmetry lowering has profound consequences on the electronic structure, namely that the bandgap changes from direct to indirect. Crucially, such symmetry-lowering configurations represent local minima in the free energy surface of the crystal and they are stabilized by van der Waals (vdW) interactions. These are the key ingredients not only for obtaining accurate lattice parameters ${ }^{2,14-17}$ (see Supplementary Fig. 1) but also for the internal geometry. Our calculations then return a picture of the $\mathrm{CH}_{3} \mathrm{NH}_{3} \mathrm{PbI}_{3}$ as a 'dynamical' bandgap semiconductor, in which the exact position of the conduction band minimum depends on the particular spatial arrangement of the molecules. Importantly, our results are robust against bandgap corrections and spin-orbit interaction $^{11,18-21}$, and deliver an absorption spectrum in good agreement with experimental data near the absorbtion edge ${ }^{7,22}$.

\section{Results}

Geometrical optimization. Let us start the discussion by presenting the relaxed crystal geometries. Initially, all the calculations have been performed at the level of the generalized gradient approximation (GGA) ${ }^{23}$ to the DFT exchange and correlation functional, including $\mathrm{vdW}$ interactions ${ }^{24}$ (see computational details section) and without explicitly considering the spinorbit coupling (SOC). Additional calculations beyond the GGA and including SOC will be discussed later in relation to the electronic structure.

In Fig. 1, the relaxed structures for the cubic phase of $\mathrm{CH}_{3} \mathrm{NH}_{3} \mathrm{PbI}_{3}$ are presented along the $x y$ and $x z$ planes for two different orientations of the $\mathrm{CH}_{3} \mathrm{NH}_{3}$ cation. Given the periodic boundary conditions, these structures effectively represent the specific case of an infinite array of perfectly aligned molecular dipoles. The relaxation process is extremely sensitive to the initial conditions. When we start the geometrical optimization with the molecular cation oriented along the (111) direction, the structure relaxes maintaining the same orientation (Fig. 1a). In contrast, by starting from a (001)-oriented molecule, the relaxation may end up with $\mathrm{CH}_{3} \mathrm{NH}_{3}$ along (011) (Fig. 1b) or an equivalent direction. However, it is important to point out that the cation energy landscape is very shallow. Indeed, both configurations represent local energy minima, with an energy difference of only about $20 \mathrm{meV}$ in favour of (011). In both cases, the lattice parameter is calculated in excellent agreement with the experimental value of $6.329 \AA$ (ref. 25) and an error of $<0.8 \%$ (see Supplementary Table 1 and Supplementary Fig. 1). If one does not include vdW forces in the relaxation, the discrepancy with the nominal value will become $>2 \%$, that is, the crystal structure is not properly described. Furthermore, the angle formed by the molecular axis and the (001) direction increases from 16 to $25^{\circ}$ on inclusion of dispersion forces. To make a well-grounded comparison with previous calculations ${ }^{11,26}$, reporting no effect of MA on the structure, we started the relaxation from the $\mathrm{X}$-ray diffraction solved structure with $\mathrm{CH}_{3} \mathrm{NH}_{3}$ along $(100)^{27}$, whose band structure is presented in Supplementary Fig. 2.

By inspecting the relaxed structures, a prominent difference between the (111) and the other two geometries appears. In fact, in the case of (011), the rotation of the cation induces a deformation and a symmetry reduction of the inorganic $\mathrm{PbI}_{6}$ octahedra. The result of such $\mathrm{PbI}_{6}$ octahedra distortion is that the $\mathrm{Pb}-\mathrm{I}$ bonds do not lie parallel to the crystal directions, but instead they slightly deviate by $\sim 6^{\circ}$. Unlike the $(111)$ case, the unit cell becomes pseudocubic with the lattice parameters along the $y$ and $z$ directions being $1 \%$ larger than that along $x$. Hence, it is clear that the dispersive forces are critical for the internal geometry optimization and consequently, as we will see next, for the electronic behaviour of hybrid perovskites.

Electronic properties. The fine differences in the crystal geometries have a qualitatively strong impact on the electronic structure. In Fig. 2, we show the electronic band structure along some high-symmetry points of the Brillouin zone calculated with and without including fully relativistic SOC. For both orientations, the energy gap computed without SOC lies in the $1.5-1.7 \mathrm{eV}$ range, close to the experimental value of about $1.55 \mathrm{eV}$ (refs 22,28). However, as already shown ${ }^{5}$, this agreement is due to a fortuitous cancellation of errors, namely the GGA underestimates the bandgap, but this is counterbalanced by the lack of the spin-orbit interaction. It is well known that the bottom of the conduction band mainly originates from the $p$ 
a
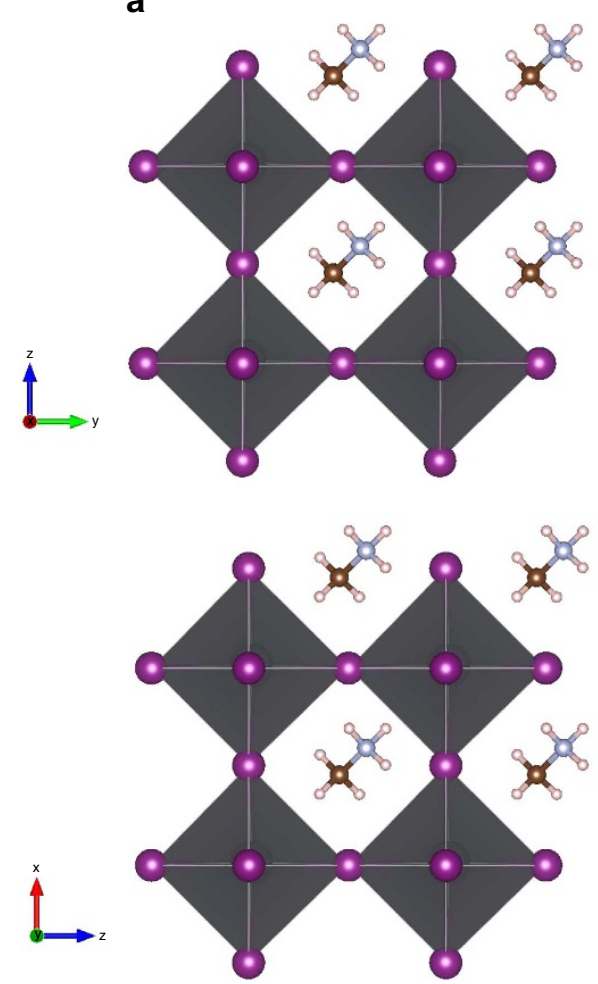

b
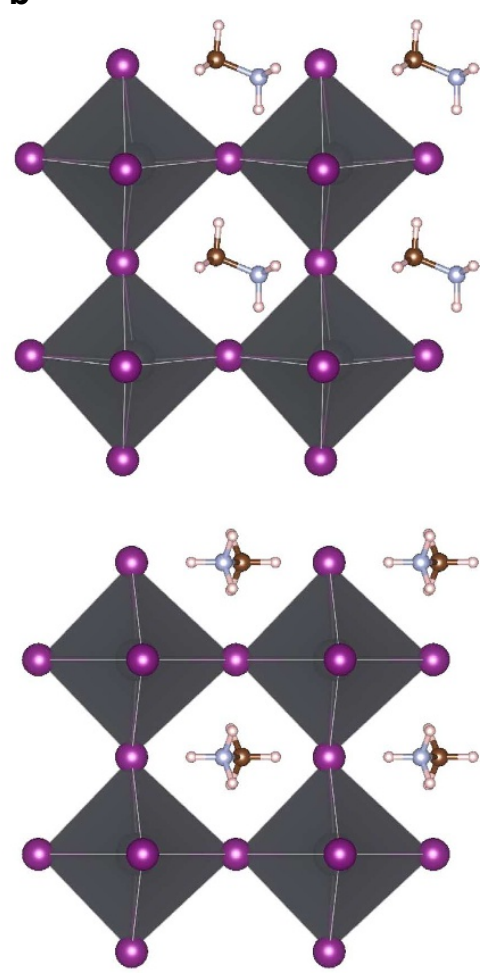

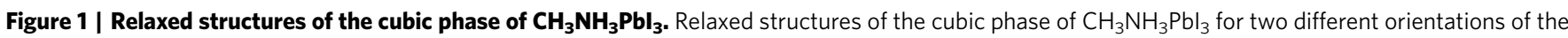
cations, namely along (a) (111) and (b) (011). (b) The views along the $x y$ and $x z$ planes are shown in the upper and lower panels, respectively.

orbitals of $\mathrm{Pb}$, while the top of the valence band is derived from the $p$ orbitals of I. The highest occupied molecular orbital of MA is found deep below the valence band, $\sim 5 \mathrm{eV}$ below the valence band maximum (VBM). Thus, one may argue that $\mathrm{CH}_{3} \mathrm{NH}_{3}$ does not play any role in the optical and electronic response of such materials, but that rather it does only contribute to their structural cohesion. However, a careful analysis of the density of states projected on the various atoms (see Fig. 3a and Supplementary Fig. 3) reveals that there is a small contribution $\sim 0.5 \mathrm{eV}$ below the VBM attributable to the organic molecules. This indicates that indeed there is an interaction between $\mathrm{CH}_{3} \mathrm{NH}_{3}$ and the inorganic $\mathrm{PbI}_{6}$ octahedra, in the form of hydrogen bonds between $\mathrm{N}$ and $\mathrm{I}$.

A closer inspection to the band structures reveals that the orientation of $\mathrm{CH}_{3} \mathrm{NH}_{3}$ has a profound impact on the nature of the bandgap. In fact, while for the (111) case the gap is direct at the R point, the cationic rotation induces a transition to an indirect gap, and when MA is along (011) the conduction band mininum (CBM) shifts along the $\mathrm{R} \rightarrow \Gamma$ line. Accordingly, the vertical energy difference between the CBM and the conduction band energy at $\mathrm{R}$ is $25 \mathrm{meV}$, as shown in the inset of Fig. 2b. The effect persists when SOC is turned on, as shown in Fig. $2 \mathrm{c}$,d. In this case, a slight shift of the VBM towards $\Gamma$ occurs as well, reducing the $\mathrm{CB}$ vertical energy difference approximately to half the non-SOC value. We stress here that the (011) orientation is not a special case-the indirect bandgap does appear for all the equivalent orientations, namely $(0 \pm 11),(01 \pm 1),( \pm 101),( \pm 110),(10 \pm 1)$ and $(01 \pm 1)$. As an example, in Supplementary Fig. 4 , we show the (0-11) case, for which the CBM shifts in the direction opposite to that shown in Fig. 2, namely $\mathrm{R} \rightarrow \mathrm{M}$.

Such a gap transformation is the most remarkable result of the interaction between the organic molecules and the inorganic framework, which is driven by the dispersive forces. As a result, $\mathrm{CH}_{3} \mathrm{NH}_{3}$ exerts strain on the $\mathrm{PbI}_{6}$ octahedra and changes the nature of the bandgap. This is unusual in the perovskite world and it is a unique peculiarity of such hybrid systems. Typical inorganic perovskites such as $\mathrm{SrTiO}_{3}$ respond to very small uniaxial strains, as those induced by the antiferrodistortive phase transition, by splitting their threefold degenerate $\mathrm{CBM}^{29}$. To the best of our knowledge, very few semiconductors see their bandgap changing from direct to indirect under the effect of strain. An example is given by layered transition metal chalcogenides, like for instance, $\mathrm{MoSe}_{2}$ single layer, where the gap change is attributed to phonon softening ${ }^{30}$. Intriguingly, also for $\mathrm{CH}_{3} \mathrm{NH}_{3} \mathrm{PbI}_{3}$, we observe some softening of our calculated low-energy optical phonon modes, when the MA molecule rotates along the (011) directions. In particular, the calculated energy red shift is of the order of $10 \mathrm{~cm}^{-1}$ (see Supplementary Fig. 5).

We have carefully verified that the indirect bandgap is not an artifact of our computation method. First, we analysed the relaxation in a $2 \times 2 \times 2$ (96 atoms) supercell to establish whether further low-symmetry configurations are possible. The supercell converged geometries are very similar to the unit cell geometries repeated in space due to periodic boundary conditions. The calculations reveal no particular changes, although the size of the supercell would allow the $\mathrm{PbI}_{6}$ octahedra to rotate or tilt. Consequently, the features of the bandgaps are preserved both at the qualitative as well as the quantitative level.

Second, we have tested different vdW functionals by comparing the geometries obtained with the Tkatchenko-Scheffler (TS) scheme $^{24}$ to those calculated with the empirical Grimme's method $^{31}$ and with a recently developed sophisticated manybody dispersion correction ${ }^{32}$. As shown in Supplementary Fig. 1, the unit cell volume compares well with the experimental value for all the vdW-corrected functionals, although that calculated with the TS scheme is closer. Thus, in general, the TS and Grimme's pairwise dispersion methods provide a sufficiently good description of our system as found in a recent work on 
molecular crystals ${ }^{17}$. In any case, the indirect bandgap is found for all the calculated geometries, including those obtained with the many-body dispersion correction. In contrast, when the dispersion interactions are not included, the gap remains direct since the structure does not present enough distortion. Then, we have performed calculations using the HSE06 (ref. 33) screened hybrid functional by starting from the relaxed structures. Also, in this case, the bandgap remains indirect (see Supplementary Fig. 3), although the overall energy distance between the CBM and the VBM increases, as expected.

Finally, we have performed additional calculations to establish whether or not the observed effect is the result of the interaction of the MA dipole with its periodic images. We have then verified that the position of the VBM and the CBM does not shift (i) on flipping the molecule's dipole moment and (ii) on applying a rigid rotation of the molecule from the 111 to the 110 orientation (Supplementary Fig. 6). This confirms that it is the distortion of the inorganic $\mathrm{PbI}_{6}$ octahedra to govern the shape of the CBM and $\mathrm{VBM}$ and not the dipole moment of the organic molecule.

As a last element of our analysis, we have investigated the relative stability of the different molecular orientations. In particular, we have performed nudged elastic band calculations and evaluated the barrier height for an MA rotation from (011) to (111), taking into account the possible changes in the cell geometry and the lattice vectors. We have found a barrier of
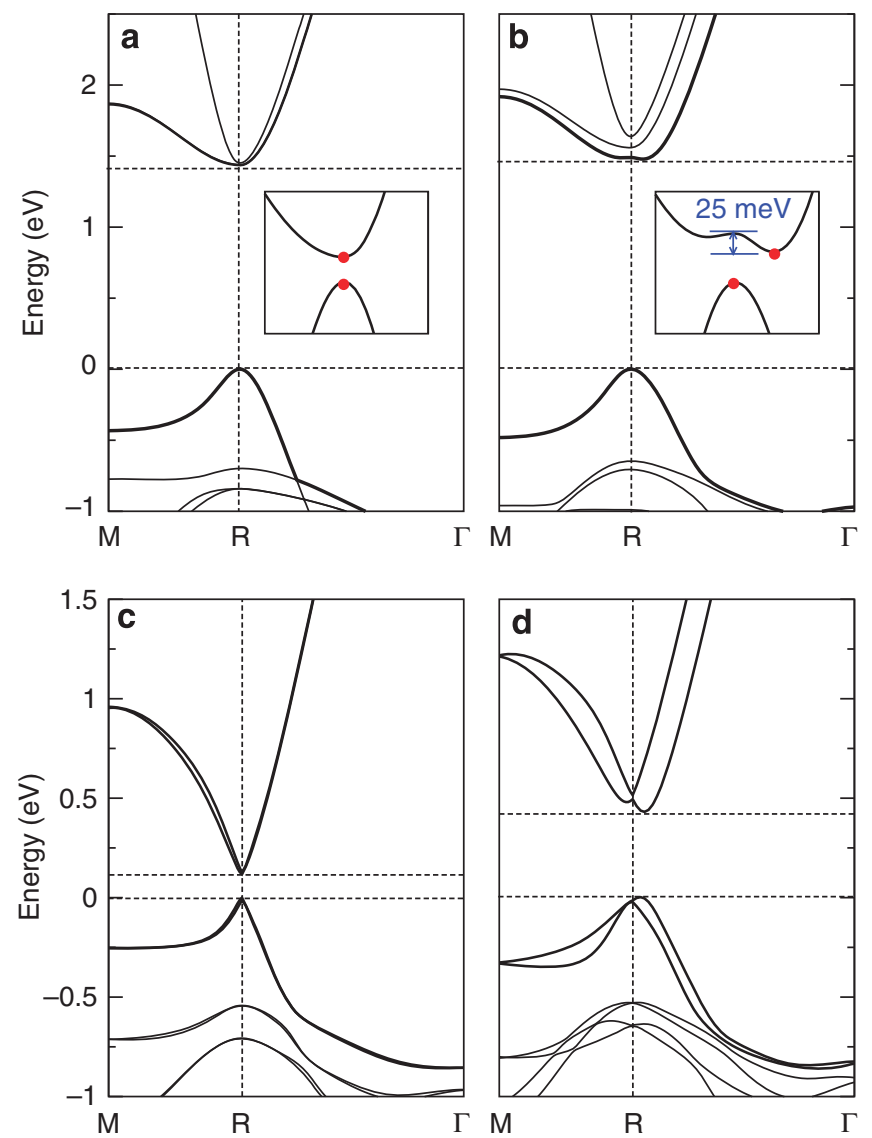

Figure 2 | Band structure of the fully relaxed $\mathrm{CH}_{3} \mathrm{NH}_{3} \mathrm{Pbl}_{3}$ crystal. The bands are shown for molecule orientations along (a) (111) and (b) (011) direction. The insets show a magnification of the bands (which have been shifted in energy for convenience) around the bandgap and highlight the changes in the VBM and CBM caused by the rotation of $\mathrm{CH}_{3} \mathrm{NH}_{3}$. Note that for the (011) orientation the bandgap becomes indirect. The relativistic SOC band structures are shown in panels (c) and (d) for (111) and (011).
$20 \mathrm{meV}\left(1.929 \mathrm{~kJ} \mathrm{~mol}^{-1}\right)$, which is less than $k_{\mathrm{B}} T$, and it is similar to values found recently in literature ${ }^{34}$. This confirms the concept that above room temperature the molecules continuously rotate. Given the strong dependence of the position in $k$-space of the $\mathrm{CBM}$ and the VBM on the cell geometry, the fast molecular rotation implies that the nature and shape of the bandgap are rapidly-varying functions of time. Recently, Mosconi et al. ${ }^{35}$ published Car-Parinello molecular dynamics simulations for a $2 \times 2 \times 2 \mathrm{CH}_{3} \mathrm{NH}_{3} \mathrm{PBI}_{3}$ supercell at $319 \mathrm{~K}$, showing fluctuations of $\pm 0.1-0.2 \mathrm{eV}$ in the position of the VBM, and thus giving evidence that the size of the bandgap changes as function of the MA rotation. Here we go a step forward and demonstrate that for some molecular orientations the bandgap turns from direct to indirect.

Optical absorption. To seek further evidence of the presence of the indirect bandgap, we have calculated the perovskite's optical absorption and compared it with available experimental data. The calculations have been performed over the electronic structure obtained with the GGA including vdW interaction in the independent particle approximation and without SOC. Although the fortuitous cancellation of errors returns a bandgap close to experiments, we concentrate here on the shape of the spectrum around the absorption edge. The calculations are performed for the two orientations of the $\mathrm{CH}_{3} \mathrm{NH}_{3}$ molecule, and the spectra are presented in Fig. 3c.

When MA is aligned along the (111) direction, the bandgap is direct and measures $1.423 \mathrm{eV}=871 \mathrm{~nm}$. The absorption profile
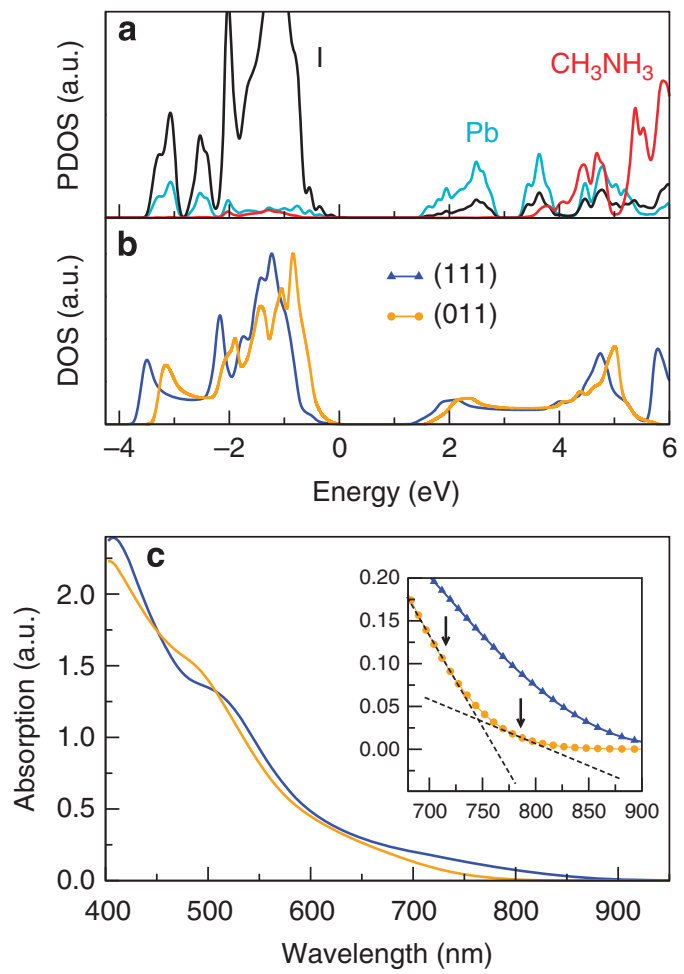

Figure 3 | Electronic and optical properties of the cubic phase

$\mathbf{C H}_{\mathbf{3}} \mathbf{N H}_{\mathbf{3}} \mathbf{P B I}_{\mathbf{3}}$. (a) Projected density of states (DOS) for the case of the (111)-oriented molecule on the $\mathrm{Pb}$ (cyan), I (black) species and the $\mathrm{CH}_{3} \mathrm{NH}_{3}$ (red). (b) DOS as a function of the chemical potential for the molecule oriented along the (111) and (011) directions are displayed. (c) The calculated absorption spectra are plotted for the different orientation of $\mathrm{CH}_{3} \mathrm{NH}_{3}$ clearly showing the impact the organic cation rotation. The inset in c is a magnification of the same quantity around the low-energy region. 
then increases monotonically from that value, indicating the possibility of direct transitions across the band edges. In contrast, when $\mathrm{CH}_{3} \mathrm{NH}_{3}$ is placed along (011) the bandgap is indirect, and in general slightly larger than that corresponding to the (111) orientation. In particular, for (011), we find a direct gap of $1.611 \mathrm{eV}=769 \mathrm{~nm}$ and an indirect one of $1.629 \mathrm{eV}=761 \mathrm{~nm}$. Here the shape of the absorption spectrum is quite different from the (111) case. We note a 'two-step' absorption profile (see the inset of Fig. 3c), with a small absorption amplitude developing at the band edge followed by a significantly more pronounced increase at around $750 \mathrm{~nm}$. This reflects the indirect nature of the bandgap and the fact that indirect transitions are not allowed, unless assisted by phonons. Certainly, the level of theory used here is not complete and one should use the many-body methods for deriving a more quantitative picture ${ }^{36}$; however, the inclusion of excitonic effects is not necessary to make our main point about the shape of the spectrum around the absorption edge. In fact, the differences between the direct and indirect bandgap are very evident from the inset of Fig. 3c, and should be compared with the typical experimental absorption spectrum measured at $300 \mathrm{~K}$ (ref. 4). This latter features a small amplitude around the bandgap followed by a rapid increase after about $50 \mathrm{~nm}$, which seems to be a robust feature in the experimentally measured spectra, regardless of the morphology of the material ${ }^{7,22,37}$ and resistent to film quality degradation ${ }^{38}$.

Whether or not such a near-edge feature can be attributed to the indirect bandgap is however difficult to establish with certainty. In fact, at $300 \mathrm{~K}$, the MAs are free to rotate and sample the many quasi-equivalent (011) and (111) orientations. Thus, it is expected that no sharp indirect bandgap can be observed, but rather a smooth absorption spectra starting around the average bandgap value. Interestingly, we point out that our finding could help explaining the recently observed line broadening in photoluminescence experiments ${ }^{39}$, attributed to coupling with phonons. In fact, low-energy phonons are associated with cation rotations, which in turn would shift the CBM to different positions. As a result, the range of optical gap would change as a function of temperature, thus broadening the emission spectrum. Measurement of the optical absorption and thermal characteristics using very high-sensitivity techniques such as photothermal deflection spectroscopy might reveal the existence of the indirect bandgap by fine lowering of temperature $<300 \mathrm{~K}$ thus freezing the MA (011)-like orientations.

\section{Discussion}

Absorbing light efficiently and transporting the photocurrent with some gained voltage are the most important aspects in the design of a solar cell. The two processes can be characterized, respectively, by an empirical absorption length, $L_{\alpha}$, inversely proportional to the absorption ${ }^{40,41}$, and by the carrier diffusion length, $L_{\text {diff. }}$ This is a measure of the mean distance travelled by carriers before recombining ${ }^{42-44}$; it is directly proportional to the carrier lifetime, $\tau$, and it depends on several factors (mobility, carrier concentration and so on). In solar cells, it is essential to fulfil the condition $r=L_{\text {diff }} / L_{\alpha}>1$ to ensure that the photogenerated carriers can be extracted and collected. For practical reasons, $r \gg 1$ is required for high power conversion efficiency $40-42,45$.

In general, direct bandgap semiconductors, such as GaAs, have strong absorption and thus short $L_{\alpha}$, but this comes with fast carrier recombination and hence relatively small $L_{\text {diff. }}$ The situation is opposite when the bandgap in indirect (for example, in $\mathrm{Si}$ ). An ideal balance between $L_{\alpha}$ and $L_{\text {diff }}$ is present in some indirect bandgap semiconductors such as $\mathrm{Cu}_{3} \mathrm{~N}$ (refs 41,42), where the direct transition starts a few $k_{\mathrm{B}} T$ above the indirect gap, yielding a short $L_{\alpha}$. The small difference between the indirect gap and the lowest direct transition ensures that the absorption is not greatly affected by the indirect gap till the direct transitions start to dominate the absorption spectrum. In contrast, the lifetime of the photogenerated carriers, which relax to the band edges in the indirect gap semiconductors, is longer than that of the direct ones by orders of magnitudes ${ }^{43}$ ensuring a large $L_{\text {diff. }}$ This class of indirect semiconductors promises remarkable improvement in efficiency via the large $L_{\text {diff }} / L_{\alpha}$ ratio $^{41,42}$.

From this short discussion and our results at hand, we can speculate on the possible origin of the high efficiency in hybrid perovskites solar cells. Our calculations establish that, under certain conditions of molecule alignment, the bandgap of the high-temperature phase of $\mathrm{CH}_{3} \mathrm{NH}_{3} \mathrm{PBI}_{3}$ can be indirect and not direct. Note that an indirect bandgap in hybrid organic/inorganic perovskite may also arise as a result of Rashba splitting ${ }^{10,46}$. This feature originates from the strain exerted on the $\mathrm{PbI}_{6}$ octahedra by the organic molecules, when these points mostly towards one of the unit cell faces rather than along the diagonal, which constitute shallow minima in the total energy profile. The indirect bandgap is tens of $\mathrm{meV}$ smaller than the direct one and the position of the CBM with respect to the VBM depends on the specific orientation of the molecules. This analysis delivers a picture of $\mathrm{CH}_{3} \mathrm{NH}_{3} \mathrm{PBI}_{3}$ as a 'dynamical' bandgap semiconductor, where the term 'dynamical' refers to the fact that the position in $k$-space of the CBM depends on the molecular alignment, which in turn fluctuates at room temperature. The calculated absorption spectrum near the band edge, characterized by a shallow amplitude at about $800 \mathrm{~nm}$ followed by a rapid enhancement within $50 \mathrm{~nm}$, is very similar for (011) and equivalent orientations of the molecules, indicating that it is a feature robust to the molecular rotation and disorder.

The presence of an indirect bandgap, with only a small displacement in $k$-space with respect to the direct point, suggests that the rapid relaxation of the photoexcited electrons to the conduction band minimum is still possible. This process is incoherent, mediated by acoustic phonons and spontaneously breaks the excitons in free carriers. As a second effect of the collapse of the electrons to the indirect conduction band minimum is that the lifetime of the minority carriers is enhanced, so that radiative recombination is partially suppressed. Such suppression is not complete since the experiments reveal luminescence; however, it contributes to prolong the carrier lifetime. In fact, it is interesting to note that experiments report that the luminescence gets suppressed as the material crystallizes $^{37}$ and that in any case it is sensitive to the sample morphology and quality ${ }^{47}$. Considering that the crystallization process leads to the geometries described here, such suppression of the luminescence on ordering supports our argument. To complete the picture, it is also worth reminding that the absence of strong scattering centres inhibits incoherent recombination, so that $L_{\text {diff }}$ remains long ${ }^{48}$.

\section{Conclusions}

Despite the tremendous advances in studying and understanding hybrid perovskites from both the theoretical and the experimental point of views, yet the reason behind the extended carrier lifetime in these materials remains a mystery. Our work suggests that the extended carrier lifetimes in $\mathrm{CH}_{3} \mathrm{NH}_{3} \mathrm{PBI}_{3}$ might be a consequence of the 'dynamical' position of the conduction band minimum forming a basin around a high-symmetry point in the $k$-space. This proposed dynamical bandgap, induced by the molecular motion, prohibits carrier recombination and favours exciton separation, thus increasing the conversion efficiency of the photvoltaic absorber $\mathrm{CH}_{3} \mathrm{NH}_{3} \mathrm{PBI}_{3}$. Crucially, all such 
phenomenology appears to be initiated by the geometry assumed by the molecules following full geometry relaxation. A set of differently oriented $\mathrm{CH}_{3} \mathrm{NH}_{3}$ cation geometries, relaxed without any symmetry constrains using DFT including vdW forces, lead to geometries where the $\mathrm{PbI}_{6}$ octahedra distort. The band structure corresponding to such geometries present an indirect bandgap, which persists on bandgap corrections via screened hybrid functionals and SOC. Finally, we propose that a possible way for engineering the efficiency of hybrid perovskites is to induce distortion to the $\mathrm{PbI}_{6}$ octahedra either by using strain or organic molecules strongly interacting with the inorganic cages.

\section{Methods}

DFT calculations. The electronic properties of the hybrid perovskites have been calculated with the all-electron FHI-aims code at the level of GGA in the PerdewBurke-Ernzerhof ${ }^{23}$ parameterization. Long-range vdW interactions have been taken into account via the TS scheme ${ }^{24}$, which is also constructed over a GGA and a pairwise dispersive potential. The reciprocal space integration was performed over an $8 \times 8 \times 8$ Monkhorst-Pack grid $^{49}$, after performing convergence tests on the total energy and the forces. A preconstructed high-accuracy all-electron basis set of numerical atomic orbitals was employed, as provided by the FHI-aims 'tight' default option. This is enough to achieve well-converged forces as confirmed by comparing the results with those obtained with the tier2 basis set. The atomic zeroth-order regular approximation was applied to treat relativistic effects. Unconstrained structural optimization was performed with the Broyden-FletcherGoldfarb-Shanno algorithm ${ }^{50}$ with with a tolerance of $10^{-3} \mathrm{eV} \AA^{-1}$.

Additional calculations, in particular, concerning the use of the hybrid functional HSE06 (ref. 33), were performed using the Gaussian suite ${ }^{51}$, with the periodic boundary condition ${ }^{52}$ code version. The Def2 (ref. 53) series of GAUSSIAN basis sets were optimized following our own procedure, described in ref. 54, for the atomic species of interest in this work. Most numerical settings in Gaussian were left at the default values, for example, uncontrained geometry optimization settings, integral cutoffs and self-consistent convergence thresholds. The standard root mean squared force threshold in Gaussian for geometry optimizations is $450 \times 10^{-6}$ hartrees per Bohr. The default $k$-point mesh of $8 \times 8 \times 8$ was used for the 12 atoms unit cell of $\mathrm{CH}_{3} \mathrm{NH}_{3} \mathrm{PbI}_{3}$.

Finally calculations involving the spin-orbit interaction have been carried out using the Vienna $a b$ initio simulation package (VASP). A plane wave basis set energy cutoff of $520 \mathrm{eV}$ was considered in the calculations and Brillouin zone integration was performed using $8 \times 8 \times 8 k$-point mesh centred at the Gamma point. Dispersion correction to the Perdew-Burke-Ernzerhof was done within the method of Tkatchenko and Scheffler (DFT-TS)-recently implemented in the 5.3.5 version of VASP - used to account for vdW interactions. The projector augmented wave pseudopotentials were used for all elements $5 d^{10} 6 s^{2} 6 p^{2}$ valence electron potential was used for the $\mathrm{Pb}$ atom. With the same set-up, VASP has been used to compute the band structures with fully relativistic SOC.

The linear optical absorption has been obtained with FHI-aims by computing the momentum matrix elements in a $5-\mathrm{eV}$ energy window around the Fermi level and then by using the Fermi golden rule approximation. A dense $k$-point sampling of $15 \times 15 \times 15$ has been employed to ensure converged spectra.

Phonon calculations. Phonon spectra were computed with the frozen-phonon approach for a fully relaxed $2 \times 2 \times 2$ supercells using the Phonopy ${ }^{55}$ code in combination with FHI-aims A displacement of 0.001 A was applied to each atom in the three space directions to compute the dynamical matrix.The phonon DOS used the $\Gamma$ point sampling, while the full phonon band structure was generated using the $\Gamma, M, X$ and $R$ high-symmetry points in the Brillouin zone.

\section{References}

1. Snaith, H. J. Perovskites: the emergence of a new era for low-cost, highefficiency solar cells. J. Phys. Chem. Lett. 4, 3623-3630 (2013).

2. Boix, P. P., Nonomura, K., Mathews, N. \& Mhaisalkar, S. G. Current progress and future perspectives for organic/inorganic perovskite solar cells. Mater. Today 17, 16-23 (2014).

3. Loi, M. A. \& Hummelen, J. C. Hybrid solar cells: perovskites under the sun. Nat. Mater. 12, 1087-1089 (2013).

4. Deschler, F. et al. High photoluminescence efficiency and optically pumped lasing in solution-processed mixed halide perovskite semiconductors. J. Phys. Chem. Lett. 5, 1421-1426 (2014).

5. Mosconi, E., Amat, A., Nazeeruddin, M. K., Grätzel, M. \& De Angelis, F. Firstprinciples modeling of mixed halide organometal perovskites for photovoltaic applications. J. Phys. Chem. C 117, 13902-13913 (2013).

6. Liu, M., Johnston, M. B. \& Snaith, H. J. Efficient planar heterojunction perovskite solar cells by vapour deposition. Nature 501, 395-398 (2013).

7. Xing, G. et al. Long-range balanced electron- and hole-transport lengths in organic-inorganic $\mathrm{CH}_{3} \mathrm{NH}_{3} \mathrm{PbI}_{3}$. Science 342, 344-347 (2013).
8. Yin, W.-J., Shi, T. \& Yan, Y. Unusual defect physics in $\mathrm{CH}_{3} \mathrm{NH}_{3} \mathrm{PbI}_{3}$ perovskite solar cell absorber. Appl. Phys. Lett. 104, 063903 (2014).

9. Kim, J., Lee, S.-H., Lee, J. H. \& Hong, K.-H. The role of intrinsic defects in methylammonium lead iodide perovskite. J. Phys. Chem. Lett. 5, 1312-1317 (2014).

10. Brivio, F., Butler, K. T., Walsh, A. \& van Schilfgaarde, M. Relativistic quasiparticle self-consistent electronic structure of hybrid halide perovskite photovoltaic absorbers. Phys. Rev. B 89, 155204 (2014).

11. Umari, P., Mosconi, E. \& De Angelis, F. Relativistic gw calculations on $\mathrm{CH}_{3} \mathrm{NH}_{3} \mathrm{PbI}_{3}$ and $\mathrm{CH}_{3} \mathrm{NH}_{3} \mathrm{SnI}_{3}$ perovskites for solar cell applications. Sci. Rep. 4, 4467 (2014).

12. Baikie, T. et al. Synthesis and crystal chemistry of the hybrid perovskite (CH3NH3) $\mathrm{PbI} 3$ for solid-state sensitised solar cell applications. J. Mater. Chem. A 1, 5628-5641 (2013).

13. Knop, O., Wasylishen, R. E., White, M. A., Cameron, T. S. \& Oort, M. J. M. V. Alkylammonium lead halides. Part 2. CH3NH3PbX3 $(\mathrm{X}=\mathrm{Cl}, \mathrm{Br}, \mathrm{I})$ perovskites: cuboctahedral halide cages with isotropic cation reorientation. Can. J. Chem. 68, 412-422 (1990).

14. Wang, Y. et al. Density functional theory analysis of structural and electronic properties of orthorhombic perovskite $\mathrm{CH}_{3} \mathrm{NH}_{3} \mathrm{PbI}_{3}$. Phys. Chem. Chem. Phys. 16, 1424-1429 (2014).

15. Menéndez-Proupin, E., Palacios, P., Wahnón, P. \& Conesa, J. C. Self-consistent relativistic band structure of the $\mathrm{CH}_{3} \mathrm{NH}_{3} \mathrm{PbI}_{3}$ perovskite. Phys. Rev. B 90, 045207 (2014)

16. Egger, D. A. \& Kronik, L. Role of dispersive interactions in determining structural properties of organic - inorganic halide perovskites: insights from first-principles calculations. J. Phys. Chem. Lett. 5, 2728-2733 (2014).

17. Kronik, L. \& Tkatchenko, A. Understanding molecular crystals with dispersioninclusive density functional theory: pairwise corrections and beyond. Acc. Chem. Res. 47, 3208-3216 (2014).

18. Even, J., Pedesseau, L., Jancu, J.-M. \& Katan, C. Importance of spinorbit coupling in hybrid organic/inorganic perovskites for photovoltaic applications. J. Phys. Chem. Lett. 4, 2999-3005 (2013).

19. Even, J., Pedesseau, L., Jancu, J.-M. \& Katan, C. DFT and KP modelling of the phase transitions of lead and tin halide perovskites for photovoltaic cells. Phys. Status Solidi RRL 8, 31-35 (2014).

20. Feng, J. \& Xiao, B. Crystal structures, optical properties, and effective mass tensors of $\mathrm{CH}_{3} \mathrm{NH}_{3} \mathrm{PbX}_{3}(\mathrm{X}=\mathrm{I}$ and $\mathrm{Br})$ phases predicted from HSE06. J. Phys. Chem. Lett. 5, 1278-1282 (2014).

21. Giorgi, G., Fujisawa, J.-I., Segawa, H. \& Yamashita, K. Cation role in structura and electronic properties of $3 \mathrm{~d}$ organic-inorganic halide perovskites: a DFT analysis. J. Phys. Chem. C 118, 12176-12183 (2014).

22. Lee, M. M., Teuscher, J., Miyasaka, T., Murakami, T. N. \& Snaith, H. J. Efficient hybrid solar cells based on meso-superstructured organometal halide perovskites. Science 338, 643-647 (2012).

23. Perdew, J. P., Burke, K. \& Ernzerhof, M. Generalized gradient approximation made simple. Phys. Rev. Lett. 77, 3865-3868 (1996).

24. Tkatchenko, A. \& Scheffler, M. Accurate molecular van der waals interactions from ground-state electron density and free-atom reference data. Phys. Rev. Lett. 102, 073005 (2009).

25. Poglitsch, A. \& Weber, D. Dynamic disorder in methylammoniumtrihalogenoplumbates (ii) observed by millimeter-wave spectroscopy. J. Chem. Phys. 87, 6373 (1987).

26. Brivio, F., Walker, A. B. \& Walsh, A. Structural and electronic properties of hybrid perovskites for high-efficiency thin-film photovoltaics from firstprinciples. APL Mater. 1, 042111 (2013).

27. Stoumpos, C. C., Malliakas, C. D. \& Kanatzidis, M. G. Semiconducting tin and lead iodide perovskites with organic cations: phase transitions, high mobilities, and near-infrared photoluminescent properties. Inorg. Chem. 52, 9019-9038 (2013).

28. Kojima, A., Teshima, K., Shirai, Y. \& Miyasaka, T. Organometal halide perovskites as visible-light sensitizers for photovoltaic cells. J. Am. Chem. Soc. 131, 6050-6051 (2009).

29. El-Mellouhi, F., Brothers, E. N., Lucero, M. J., Bulik, I. W. \& Scuseria, G. E. Structural phase transitions of the metal oxide perovskites SrTiO3, LaAlO3, and LaTiO3 studied with a screened hybrid functional. Phys. Rev. B 87, 035107 (2013).

30. Horzum, S. et al. Phonon softening and direct to indirect band gap crossover in strained single-layer MoSe2. Phys. Rev. B 87, 125415 (2013).

31. Grimme, S. Semiempirical GGA-type density functional constructed with a long-range dispersion correction. J. Comput. Chem. 27, 1787-1799 (2006).

32. Tkatchenko, A., DiStasio, R. A., Car, R. \& Scheffler, M. Accurate and efficient method for many-body van der Waals interactions. Phys. Rev. Lett. 108, 236402 (2012).

33. Krukau, A. V., Vydrov, O. A., Izmaylov, A. F. \& Scuseria, G. E. Influence of the exchange screening parameter on the performance of screened hybrid functionals. J. Chem. Phys. 125, 224106 (2006).

34. Frost, J. M. et al. Atomistic origins of high-performance in hybrid halide perovskite solar cells. Nano Lett. 14, 2584-2590 (2014). 
35. Mosconi, E., Quarti, C., Ivanovska, T., Ruani, G. \& De Angelis, F. Structural and electronic properties of organo-halide lead perovskites: a combined IRspectroscopy and ab initio molecular dynamics investigation. Phys. Chem. Chem. Phys. 16, 16137-16144 (2014).

36. Even, J., Pedesseau, L. \& Katan, C. Analysis of multivalley and multibandgap absorption and enhancement of free carriers related to exciton screening in hybrid perovskites. J. Phys. Chem. C 118, 11566-11572 (2014).

37. Burschka, J. et al. Sequential deposition as a route to high-performance perovskite-sensitized solar cells. Nature 499, 316-319 (2013).

38. Niu, G. et al. Study on the stability of $\mathrm{CH}_{3} \mathrm{NH}_{3} \mathrm{PbI}_{3}$ films and the effect of postmodification by aluminum oxide in all-solid-state hybrid solar cells. J. Mater. Chem. A 2, 705-710 (2014).

39. Wehrenfennig, C., Liu, M., Snaith, H. J., Johnston, M. B. \& Herz, L. M. Homogeneous emission line broadening in the organo lead halide perovskite CH3NH3PbI3XClX. J. Phys. Chem. Lett. 5, 1300-1306 (2014).

40. Loferski, J. J. Theoretical considerations governing the choice of the optimum semiconductor for photovoltaic solar energy conversion. J. Appl. Phys. 27, 777-784 (1956).

41. Alharbi, F. et al. Abundant non-toxic materials for thin film solar cells: Alternative to conventional materials. Renew. Energ. 36, 2753-2758 (2011).

42. Zakutayev, A. et al. Defect tolerant semiconductors for solar energy conversion. J. Phys. Chem. Lett. 5, 1117-1125 (2014).

43. Fonash, S. Solar Cell Device Physics (Academic Press, 2010).

44. Law, M., Solley, E., Liang, M. \& Burk, D. Self-consistent model of minoritycarrier lifetime, diffusion length, and mobility. IEEE Electron. Device Lett. 12, 401-403 (1991).

45. Grätzel, M. Photoelectrochemical cells. Nature 414, 338-344 (2001)

46. Stroppa, A. et al. Tunable ferroelectric polarization and its interplay with spin - orbit coupling in tin iodide perovskite. Nat. Commun. 5, 5900 (2014).

47. Choi, J. J., Yang, X., Norman, Z. M., Billinge, S. J. L. \& Owen, J. S. Structure of methylammonium lead iodide within mesoporous titanium dioxide: active material in high-performance perovskite solar cells. Nano Lett. 14, 127-133 (2013).

48. Stranks, S. D. et al. Electron-hole diffusion lengths exceeding 1 micrometer in an organometal trihalide perovskite absorber. Science 342, 341-344 (2013).

49. Monkhorst, H. J. \& Pack, J. D. Special points for brillouin-zone integrations. Phys. Rev. B 13, 5188-5192 (1976).

50. Press, W. H., Teukolsky, S. A., Vetterling, W. T. \& Flannery, B. P. Numerical Recipes 3rd edn (Cambridge Univ. Press, 1997).

51. Frisch, M. J. et al. Gaussian 09 Revision $\{B\} .01$ (Gaussian Inc., 2009).

52. Kudin, K. N. \& Scuseria, G. E. Linear-scaling density-functional theory with gaussian orbitals and periodic boundary conditions: efficient evaluation of energy and forces via the fast multipole method. Phys. Rev. B 61, 16440-16453 (2000).
53. Weigend, F. \& Ahlrichs, R. Balanced basis sets of split valence, triple zeta valence and quadruple zeta valence quality for $\mathrm{H}$ to $\mathrm{Rn}$ : design and assessment of accuracy. Phys. Chem. Chem. Phys. 7, 3297-3305 (2005).

54. El-Mellouhi, F., Brothers, E. N., Lucero, M. J. \& Scuseria, G. E. Modeling of the cubic and antiferrodistortive phases of $\mathrm{SrTiO}_{3}$ with screened hybrid density functional theory. Phys. Rev. B 84, 115122 (2011).

55. Togo, A., Oba, F. \& Tanaka, I. First-principles calculations of the ferroelastic transition between rutile-type and $\mathrm{CaCl}_{2}$-type $\mathrm{SiO}_{2}$ at high pressures. Phys. Rev B 78, 134106 (2008)

\section{Acknowledgements}

This work is sponsored by the European Research Council, Quest project, (C.M. and S.S.) and by the Qatar Environment and Energy Research Institute (F.E.-M., F.A., N.T. and S.K.). Computational resources have been provided by the supercomputer facilities at the Trinity Center for High Performance Computing, at ICHEC (project tcphy038b) and the research computing at Texas A\&M University at Qatar. We acknowledge fruitful discussions with Simon Gelinas, Marcelo Carignano, Mohamed Lamine Madjet and Felix Deschler.

\section{Author contributions}

C.M. and F.E.-M. contributed equally to the manuscript. C.M. and F.E.-M. performed the computational research, C.M., F.E.-M., S.K., N.T., F.A. and S.S. discussed the results and contributed to the preparation of the manuscript.

\section{Additional information}

Supplementary Information accompanies this paper at http://www.nature.com/ naturecommunications

Competing financial interests: The authors declare no competing financial interests.

Reprints and permission information is available online at http://npg.nature.com/ reprintsandpermissions/

How to cite this article: Motta, C. et al. Revealing the role of organic cations in hybrid halide perovskite $\mathrm{CH}_{3} \mathrm{NH}_{3} \mathrm{PbI}_{3}$. Nat. Commun. 6:7026 doi: 10.1038/ncomms8026 (2015).

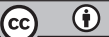

This work is licensed under a Creative Commons Attribution 4.0 International License. The images or other third party material in this article are included in the article's Creative Commons license, unless indicated otherwise in the credit line; if the material is not included under the Creative Commons license, users will need to obtain permission from the license holder to reproduce the material. To view a copy of this license, visit http://creativecommons.org/licenses/by/4.0/ 